\title{
A Comparison of Cancer Detection Rates Between Template Systematic Biopsies Obtained Using Magnetic Resonance Imaging-Ultrasound Fusion Machine and Freehand Transrectal Ultrasound-Guided Systematic Biopsies
}

\author{
Connor Hoge, BS, Sean Maynor, MPH, ${ }^{1}$ Tianyuan Guan, MPH, ${ }^{2}$ Rand Naffouje, MD, ${ }^{3}$ Marepalli Rao, PhD, \\ Sadhna Verma, MD, ${ }^{4}$ and Abhinav Sidana, $\mathrm{MD}^{1}$
}

\begin{abstract}
Introduction: There are reports that the 12-core template systematic biopsies ( $\mathrm{SBx}$ ) obtained by using software registration machines (e.g., Artemis) have higher cancer detection rates (CDRs) of prostate cancer (PCa) than the standard, freehand 12-core transrectal ultrasound (TRUS)-guided biopsies. The goal of our study is to compare the clinically significant (CS) CDRs of SBx in two independent cohorts who underwent freehand TRUS-SBx alone (Cohort A) or machine-guided SBx as part of a combined MRI-ultrasound (MRI-US) fusion biopsy (FBx) (Cohort B).

Materials and Methods: A retrospective review of all patients undergoing prostate biopsies over a 4-year period at the University of Cincinnati Medical Center was performed. CS cancer was defined as having a Gleason score 27. MRI-US FBx were obtained by using an Artemis software registration device (ARTEMIS ${ }^{\mathrm{TM}}$, Eigen, Inc., Grass Valley, CA). Statistical significance was considered at $p<0.05$.

Results: Nine hundred and thirty men underwent SBx (Cohort A: 474, Cohort B: 456). There were no statistical differences between cohort A and B in CS CDRs in the overall population (39.3\% vs 33.8\%; $p=0.093)$, biopsy naive patients $(40.4 \%$ vs $39.8 \% ; p=0.951)$, or patients with a prior negative biopsy $(22.7 \%$ vs $25.0 \%$; $p=0.910$ ). Multivariate logistic regression controlling for age, race, prostate-specific antigen level, prostate volume, abnormal digital rectal exam, and family history of PCa demonstrated comparable CS CDRs, which was maintained when further stratified by prior biopsy history (all patients: odds ratio [OR] 0.99, 95\% confidence interval [CI] 0.71-1.38, $p=0.958$; biopsy naive: OR 0.79, 95\% CI 0.51-1.22, $p=0.291$; prior negative biopsy: OR $0.64,95 \%$ CI $0.21-1.75, p=0.403$ ).

Conclusions: Our study did not find a significant difference in the CS CDRs of machine-guided SBx compared with the freehand TRUS-SBx. Unless the SBx is done at the time of FBx, the use of these machines for obtaining SBx only is unlikely to result in any increase of CS CDRs.
\end{abstract}

Keywords: fusion biopsy, multiparametric MRI-US fusion biopsy, systematic biopsy, prostate cancer, transrectal ultrasound

\section{Introduction}

T HE STANDARD OF care for the diagnosis of prostate cancer (PCa) is a 12-core transrectal ultrasound (TRUS)guided systematic biopsy (SBx). The standard TRUS-SBx is a handheld procedure, where 12 random cores are taken from the prostate. This method is highly operator-dependent and is systematically poor at sampling certain areas such as the peripheral zone and anterior portion of the prostate, leading to low values of sensitivity for cancer detection rates (CDRs) of SBx. ${ }^{1}$

Multiparametric magnetic resonance imaging (mpMRI) fusion biopsy (FBx) has been found to better detect clinically significant (CS) PCa, while decreasing the detection of

\footnotetext{
${ }^{1}$ Division of Urology, Department of Surgery, ${ }^{2}$ Division of Biostatistics and Bioinformatics, ${ }^{3}$ Division of Hematology and Oncology, ${ }^{4}$ Department of Radiology, and University of Cincinnati, Cincinnati, Ohio, USA.

Abstract presented at the 93rd Annual North Central Section of the American Urological Association. 2019 September 13; Chicago, IL, USA.
} 
insignificant disease as compared with the standard, 12-core TRUS-guided biopsy. ${ }^{2,3}$ During such biopsy, a TRUS of the prostate is obtained in real time and combined with the mpMRI image by using a software-assisted (fusion) registration device, creating a 3-dimensional (3D) model of the prostate. In addition to obtaining cores from the targeted area seen as an abnormality on mpMRI, 12 random cores are typically taken with the software registration device, similar to the standard, freehand systematic 12-core TRUS-SBx. The systematic template in these machines utilizes an array of 12 pre-determined locations on the prostate (base, middle, and apex of the left lateral, left medial, right medial, and right lateral regions of the prostate), which allows appropriate spacing and ensures the cores are not clustered together. ${ }^{4}$

Understandably, most of the research with FBx focuses on the targeted or targeted combined with the systematic portion of the biopsy rather than the systematic portion taken with the assistance of the machine registered template. It is unknown whether the SBx obtained by using these templates would be more accurate in the detection of CS PCa than the standard, freehand TRUS-SBx.

To our knowledge, this is the first study looking at the CDRs of the systematic arm of the machine registered template software used in FBx devices. This study seeks to determine whether the machine-guided SBx is superior to the conventional 12-core freehand TRUS-SBx in the detection of CS PCa.

\section{Materials and Methods}

\section{Study population}

Our study was approved by the University of Cincinnati institutional review board. We retrospectively reviewed all patients who underwent either SBx alone or in combination with an FBx between January 2014 and December 2018 at our institution. Patients with a prostate-specific antigen (PSA) level $>20 \mathrm{ng} / \mathrm{mL}$ were excluded, as well as patients with a history of $\mathrm{PCa}$, resulting in a final cohort of 930 patients for analysis.

\section{Study data}

Patient demographic, clinical, and pathological data were recorded. CS cancer was defined as a Gleason score (GS) $\geq 7$. Histopathological results of the prostate biopsy were further categorized into the following five groups: low-risk disease as a GS of $3+3$ or benign (Group 1), intermediate-risk disease as a GS of $3+4$ (Group 2) or a GS of $4+3$ (Group 3), and high-risk disease as a GS of 8 (Group 4) or a GS of 9 and 10 (Group 5). The total cohort of 930 patients was further subdivided into biopsy naive patients (600) and patients with a history of a prior negative biopsy (217) for analysis.

\section{Prostate biopsy}

Prostate biopsies were performed as a single session of either standard, 12-core freehand TRUS-SBx or a machine-guided SBx as part of an FBx device (ARTEMIS ${ }^{\mathrm{TM}}$, Eigen, Inc., Grass Valley, CA). The Artemis machine-registration device incorporates a semi-robotic mechanical arm with electronic encoders that is used to scan, digitize, track, and more precisely navigate prostate needles. An MRI of the prostate was obtained, and suspicious lesions were identified by a board-certified ra- diologist. This image was fused with a live, ultrasound-guided image of the prostate and used to target the suspicious lesions. Targeted cores were taken from these identified lesions, followed by 12 cores by using a machine-guided template that allows for appropriate spacing of the random cores and ensures a more consistent sampling of the prostate.

\section{Statistical analysis}

Statistical analysis was performed by using the computing software R 3.62 (R Core Team, 2017). Two-sample Wilcoxon rank-sum test was used to compare medians of continuous variables. Pearson Chi-square test and Fisher's exact test were used to compare proportions of categorical variables. CDRs of CS cancer were compared in two independent cohorts, wherein patients underwent freehand TRUS-SBx alone (Cohort A) or machine-guided SBx as part of a combined MRI-ultrasound $\mathrm{FBx}$ by using a software registration machine (Cohort B). Further subgroup analysis was done on biopsy naive patients and patients with a prior negative biopsy history.

A multivariate logistic regression model was prepared to control for possible confounding clinical and demographic variables, with CS CDR as the response variable. Statistical significance was defined as a two-sided $p$-value of $<0.05$.

\section{Results \\ Patient demographics}

Nine-hundred thirty patients with a history of SBx for suspicion of PCa were included in this study (474 in Cohort A and 456 in Cohort B). Median age (interquartile range [IQR]) and PSA level (IQR) were 64.5 (59.0-70.0) years and 6.4 (5.0-9.1) $\mathrm{ng} / \mathrm{mL}$ in Cohort $\mathrm{A}$, respectively. In Cohort $\mathrm{B}$, median age (IQR) and PSA (IQR) were 65.0 (60.0-70.0) years and 6.8 (5.1-9.7) ng/mL, respectively. The median number of cores taken was 12 (12-12) in Cohort A and 12 (12-13) in Cohort B. The biopsy-naive number of patients (\%) was 409 $(86.3 \%)$ in Cohort A and 191 (42.1\%) in Cohort B. History of prior negative biopsy occurred in $45(9.5 \%)$ patients in Cohort A and in $172(52.0 \%)$ patients in Cohort B. Table 1 summarizes patient demographics with biopsy results.

\section{Cancer detection rate}

A total of 184 patients (39.3\%) in Cohort A and 154 (33.8\%) in Cohort B were found to have CS cancer. Among the subset of biopsy naive patients, there were 165 patients with CS cancer in Cohort A $(40.4 \%)$ and 76 patients $(38.0 \%)$ in Cohort B $(p=0.951)$. Within the subset of patients with a prior negative biopsy, there were 10 patients $(22.7 \%)$ in Cohort A and 43 patients $(25.0 \%)$ in Cohort B who were found to have CS disease ( $p=0.907)$. Predictors such as age, PSA, abnormal digital rectal exam (DRE), race (black), and prostate volume were found to be significant predictors of CS CDR in each of the populations (Table 2).

On multivariate logistic regression analysis after adjusting for age, PSA, DRE, race, prostate volume, and family history of $\mathrm{PCa}$, the type of SBx was not a significant predictor for CS CDR (odds ratio [OR] 0.99; 95\% confidence interval [CI] $0.71-1.38, p=0.958)$. The results were similar on regression analysis stratified by the biopsy naive (OR 0.79; $95 \%$ CI $0.51-1.22 ; p=0.291$ ) or prior negative biopsy history (OR 0.64 ; 95\% CI 0.21-1.75; $p=0.403$ ) (Table 3). 
Table 1. Clinical, Demographic, and Pathologic Data of the Entire Cohort

\begin{tabular}{|c|c|c|c|}
\hline & $\begin{array}{c}\text { Cohort A } \\
\text { Freehand SBx } \\
\mathrm{n}=474\end{array}$ & $\begin{array}{c}\text { Cohort B } \\
\text { Machine-guided SBx } \\
\mathrm{n}=456\end{array}$ & $\mathrm{p}$ \\
\hline Median age (IQR) & $64.5(59.0-70.0)$ & $65(60.0-70.0)$ & 0.27 \\
\hline Race $(\%)$ & & & $<0.001$ \\
\hline White & $275(58.0)$ & $363(80.1)$ & \\
\hline Black & $182(38.4)$ & 77 (17.0) & \\
\hline Other & $17(3.6)$ & $13(2.9)$ & \\
\hline Median prostate volume cc (IQR) & $43.9(32.8-48.0)$ & $50.6(37.2-69.2)$ & $<0.001$ \\
\hline Positive family history PCa (\%) & 88 (18.6) & $125(29.3)$ & $<0.001$ \\
\hline Median PSA ng/mL (IQR) & $6.4(5.0-9.1)$ & $6.8(5.1-9.7)$ & 0.26 \\
\hline Abnormal DRE (\%) & $102(21.6)$ & $44(10.5)$ & $<0.001$ \\
\hline Biopsy naïve & $409(86.3)$ & $191(42.1)$ & $<0.001$ \\
\hline Prior negative biopsy & $45(9.5)$ & $172(52.0)$ & $<0.001$ \\
\hline Median no. of cores (IQR) & $12(12-12)$ & $12(12-13)$ & 0.135 \\
\hline \multicolumn{4}{|l|}{ CS cancer detection rate $(\%)$} \\
\hline All & $184(39.3)$ & $154(33.8)$ & 0.093 \\
\hline Biopsy naïve & $165(40.4)$ & $76(39.8)$ & 0.951 \\
\hline Prior negative biopsy & $10(22.7)$ & $43(25.0)$ & 0.907 \\
\hline \multicolumn{4}{|l|}{ Gleason group (\%) } \\
\hline Group 1 & $284(60.7)$ & $302(66.23)$ & \\
\hline Group 2 & $103(22.0)$ & $85(18.64)$ & \\
\hline Group 3 & $37(7.9)$ & $32(7.02)$ & \\
\hline Group 4 & $21(4.49)$ & $23(5.04)$ & \\
\hline Group 5 & $23(4.91)$ & $14(3.07)$ & \\
\hline
\end{tabular}

$\mathrm{CS}=$ clinically significant; $\mathrm{DRE}=$ digital rectal exam; $\mathrm{IQR}=$ interquartile range; $\mathrm{PCa}=$ prostate cancer; $\mathrm{PSA}=$ prostate-specific antigen; $\mathrm{SBx}=$ systematic biopsy.

\section{Discussion}

The continual development and increased utilization of FBx has led to a shift in the way PCa is diagnosed. Many studies have shown that the utility of FBx is in the combination of SBx and MRI-targeted cores, ${ }^{3-8}$ although some studies argue that MRI-targeted cores alone would significantly reduce insignificant cancer diagnosis. ${ }^{9}$ In contrast to the multitude of studies on the possible efficacy of MRItargeted biopsy alone in FBx, to our knowledge, there has not been a study that looked solely at utilization of the machineguided SBx of an FBx captured by a software-assisted (fusion) registration device. We sought to compare the CS CDRs of machine-guided SBx with the standard, freehand TRUS-

TABle 2. Multivariate Logistic Regression Analysis Detecting Clinically Significant Cancer in All Patients After Controlling FOR THE INDIVIDUAL VARIABLES AND BIOPSY MODALITY

\begin{tabular}{lcc}
\hline All patients $(\mathrm{n}=930)$ & & \\
\hline & & $\mathrm{p}$ \\
\hline Age $(95 \%$ CI $)$ & $<0.001$ \\
PSA & $1.07(1.04-1.09)$ & $<0.001$ \\
Race & $1.14(1.09-1.19)$ & \\
$\quad$ Black & $1.20(0.83-1.72)$ & 0.338 \\
$\quad$ Other & $0.32(0.09-0.94)$ & 0.055 \\
Prostate volume & $0.96(0.95-0.97)$ & $<0.001$ \\
Positive family history & $1.11(0.76-1.62)$ & 0.577 \\
Abnormal DRE & $1.94(1.27-2.98)$ & 0.002 \\
\hline
\end{tabular}

$\mathrm{CI}=$ confidence interval; $\mathrm{OR}=$ odds ratio.
SBx in hopes of identifying a newfound usage for softwareassisted (fusion) registration devices in the detection of PCa.

Our results show that there were no significant differences in the CS CDRs between the freehand TRUS-SBX and machine-guided SBx acquired by software-assisted registration devices. Further, analysis within biopsy naive patients and patients with a prior negative biopsy history yielded no significant differences. After controlling for possible confounding variables such as age, PSA, race, family history of $\mathrm{PCa}, \mathrm{DRE}$ and prostate volume, there remained no significant differences in the CS CDR between the two cohorts.

Given the high probability of random and systematic errors in freehand TRUS-SBx, ${ }^{1}$ it was our thought that the machineguided SBx would allow for more uniform, accurate sampling of the prostate and increase CS CDRs. However, our results found no significant differences in the CS CDRs between the two SBx modalities. The machine-guided SBx does have the advantage of creating a 3D model of the prostate compared with the 2-dimensional image used in a

Table 3. Multivariate Logistic Regression ANALYSIS OF FREEHAND TRUS-SBX DETECTING CS CANCER Over MaChINE-Guided SBX After Controlling for Age, Race, PSA, Abnormal DRE, Prostate Volume, and Family History of PCA

\begin{tabular}{lcc}
\hline & OR $(95 \%$ CI $)$ & $\mathrm{p}$ \\
\hline All patients & $0.99(0.71-1.38)$ & 0.958 \\
Biopsy naïve & $0.79(0.51-1.22)$ & 0.291 \\
Prior negative biopsy & $0.64(0.21-1.75)$ & 0.403 \\
\hline
\end{tabular}

TRUS $=$ transrectal ultrasound. 
freehand TRUS-SBX and records the location of cores taken from the prostate, but it still relies on random cores taken from the prostate.

Given that both of the biopsy modalities sampled 12 random cores from the prostate, it is possible that the number of random cores sampled from the prostate is what dictates the CDR. Scattoni and colleagues ${ }^{10}$ found that the 18 -core prostate biopsy detects a significantly higher number of cancers compared with the standard 12-core biopsy in patients with a prostate volume $>55 \mathrm{cc}$. Ploussard and colleagues ${ }^{11}$ compared CDRs in 2753 patients, finding CDRs using sextant, 12-core, and 21core schemes of $32.5 \%, 40.4 \%$, and $43.3 \%$, respectively. They found that the 21-biopsy scheme improved the CDR by $6.7 \%$ overall $(p<0.001)$ without significantly increasing the rate of insignificant PCa detected compared with the 12-core scheme $(p=0.503)$. However, the current recommendations from the American Urological Association conclude that only limited evidence supports the use of biopsy schemes over 12 cores. ${ }^{12}$

There are several limitations to our study. First, it is a single institution study. Stronger conclusions could be drawn from a larger, multi-institutional study. Second, the potential for selection bias and a possible lack of powered analysis associated with the retrospective nature of the study must be noted. Lastly, we had five different providers participating in the study with various levels of training and experience, some of which only performed freehand TRUS-SBx. Nonetheless, we believe our single institution study on the comparison of CS CDRs between machineguided SBX and freehand TRUS-SBX is valid and completes the objectives of the study.

\section{Conclusions}

Following trends of increased usage of FBx via software registration devices in the detection of $\mathrm{PCa}$, this study was the first of its kind to compare machine-guided SBx at the time of FBx vs the standard freehand TRUS-SBx. The results from our single institution study demonstrate that machine-guided SBx did not detect more CS disease compared with freehand TRUS-SBx.

\section{Author Disclosure Statement}

No competing financial interests exist.

\section{Funding Information}

This study neither received nor required any sources of funding.

\section{References}

1. Giganti F, Moore CM. A critical comparison of techniques for MRI-targeted biopsy of the prostate. Transl Androl Urol 2017;6:432-443.

2. Sonn GA, Chang E, Natarajan S, et al. Value of targeted prostate biopsy using magnetic resonance-ultrasound fusion in men with prior negative biopsy and elevated prostatespecific antigen. Eur Urol 2014;65:809-815.

3. Sidana A, Watson MJ, George AK, et al. Fusion prostate biopsy outperforms 12-core systematic prostate biopsy in patients with a prior negative systematic biopsy: A multiinstitutional analysis. Urol Onc 2018;36:341.e1-e341.e7.

4. Kongnyuy M, George AK, Rastinehad A, et al. Magnetic resonance imaging-ultrasound fusion-guided prostate bi- opsy: Review of technology, techniques and outcomes Curr Urol Rep 2016;17:32.

5. Ahmed HU, El-Shater Bosaily A, Brown LC, et al. Diagnostic accuracy of multi-parametric MRI and TRUS biopsy in prostate cancer (PROMIS): A paired validating confirmatory study. Lancet 2017;389:815-822.

6. Kasivisvanathan V, Rannikko AS, Borghi M, et al. MRItargeted or standard biopsy for prostate-cancer diagnosis. N Engl J Med 2018;378:1767-1777.

7. Panebianco V, Barchetti F, Sciarra A, et al. Multiparametric magnetic resonance imaging vs. standard care in men being evaluated for prostate cancer: A randomized study. Urol Oncol 2015;33:17.e1-17.e7.

8. Hoge C, Verma S, Lama DJ, et al. Racial disparity in the utilization of multiparametric MRI-ultrasound fusion biopsy for the detection of prostate cancer. Prostate Cancer Prostatic Dis 2020 [Epub ahead of print]; DOI: 10.1038/s41391-0200223-5.

9. Elwenspoek MMC, Sheppard AL, McInnes MDF, et al. Comparison of multiparametric magnetic resonance imaging and targeted biopsy with systematic biopsy alone for diagnosis of prostate cancer. JAMA Network Open 2019;2 e198427.

10. Scattoni V, Roscigno M, Raber M, et al. Initial extended transrectal prostate biopsy-Are more prostate cancer detected with 18 cores than with 12 cores? J Urol 2008;179: 1327-1331.

11. Ploussard G, Nicolaiew N, Marchand C, et al. Prospective evaluation of an extended 21-core biopsy scheme as initial prostate cancer diagnostic strategy. Eur Urol 2014;65:154-161.

12. Taneja SSBM, Carter HB, Schellhammer P, et al. White Paper: AUA/Optimal Techniques of Prostate Biopsy and Specimen Handling, 2013. www.auanet.org/common/pdf/ education/clinicalguidance/Prostate-Biopsy-WhitePaper.pdf (Accessed March 2, 2020).

Address correspondence to: Abhinav Sidana, $M D$ Division of Urology Department of Surgery University of Cincinnati School of Medicine 231 Albert Sabin Way ML 0589 Cincinnati, $\mathrm{OH} 45267$

USA

E-mail: abhinav.sidana@uc.edu

$$
\begin{aligned}
& \text { Abbreviations Used } \\
& \text { CDRs }=\text { cancer detection rates } \\
& \mathrm{CI}=\text { confidence interval } \\
& \mathrm{CS}=\text { clinically significant } \\
& 3 \mathrm{D}=3 \text {-dimensional } \\
& \mathrm{DRE}=\text { digital rectal exam } \\
& \mathrm{FBX}=\text { fusion biopsy } \\
& \mathrm{GS}=\text { Gleason score } \\
& \mathrm{IQR}=\text { interquartile range } \\
& \mathrm{OR}=\text { odds ratio } \\
& \mathrm{PCa}=\text { prostate cancer } \\
& \mathrm{PSA}=\text { prostate-specific antigen } \\
& \mathrm{SBX}=\text { systematic biopsy } \\
& \mathrm{TRUS}=\text { transrectal ultrasound }
\end{aligned}
$$

